\title{
3D CURRENT ALGEBRA AND TWISTED K THEORY
}

\author{
JOUKO MICKELSSON
}

\begin{abstract}
Equivariant twisted K theory classes on compact Lie groups $G$ can be realized as families of Fredholm operators acting in a tensor product of a fermionic Fock space and a representation space of a central extension of the loop algebra $L G$ using a supersymmetric Wess-Zumino-Witten model. The aim of the present article is to extend the construction to higher loop algebras using an abelian extension of a $3 D$ current algebra. We have only partial success: Instead of true Fredholm operators we have formal algebraic expressions in terms of the generators of the current algebra and an infinite dimensional Clifford algebra. These give rise to sesquilinear forms in a Hilbert bundle which transform in the expected way with respect to $3 D$ gauge transformations but do not define true Hilbert space operators.
\end{abstract}

\section{INTRODUCTION}

I have warm memories from many occasions I had the opportunity to meet Ludwig Faddeev, mostly in Helsinki and Stockholm. At one point we had very close mathematical interests although we did not have direct co-operation on the subject. This contribution to Faddeev's memorial volume is closely related to that topic. I was working during 1983 on the problem how an additional ChernSimons term in the Yang-Mills action affects the current algebra commutation relations through an abelian extension of the current algebra; the article came out as preprint in the fall 1983, published later as [5]. I met Ludwig next spring in Helsinki and I understood that he had been thinking about the same problem and in fact he had just completed an article with Samson Shatasvili [8] with similar results. In this contribution the abelian extensions of current algebras which we found at that time play a central role.

Twisted K theory on compact Lie groups has been discussed from several points of view in the past. First, let us recall some basic definitions. Let $P U(H)=$ $U(H) / S^{1}$ be the projective unitary group of a complex Hilbert space $H$. A principal bundle over a space $X$ with fiber $P U(H)$ is defined up to equivalence by a cohomology class $\Omega \in \mathrm{H}^{3}(X, \mathbb{Z})$, called the Dixmier-Douady class. The group $P U(H)$ acts by conjugation on the space of Fredholm operators in $H$. This action defines a bundle over $X$ with fiber given by the space of Fredholm operators. The homotopy classes of sections of this fiber bundle define the twisted $\mathrm{K}$ theory $\mathrm{K}^{*}(X, \Omega)$. This cohomology is $\mathbb{Z}_{2}$ graded, the even part corresponds to all Fredholm operators on $H$ and the odd part to self-adjoint Fredholm operators with essential positive and negative spectrum.

In the case when $X=G$ is a compact Lie group one is also interested in the equivariant twisted classes, $\mathrm{K}_{G}^{*}(G, \mathbb{Z})$, where the $G$ action on itself is given by conjugation. The nonequivariant case is solved for compact simple Lie groups in [4. The equivariant classes can be explicitly constructed using a supersymmetric 
conformal field theory model (Wess-Zumino-Witten model) in $1+1$ space time dimensions [3]. More detailed discussion can be found in [2] including the proof of completeness of the construction.

The construction in the equivariant case is related to the fact that the compact Lie group is actually the moduli space of gauge connections on the unit circle $S^{1}, G=\mathcal{A} / \mathcal{G}_{0}$ where $\mathcal{A}$ is the space of smooth gauge connections over $S^{1}$ and $\mathcal{G}_{0}$ denotes the group of based gauge transformations. Thus it is natural to ask if the construction can be generalized to higher dimensions, to the case of gauge potentials on a vector bundle over a compact spin manifold. In this article we shall take the modest step and study the case of the 3 -dimensional torus $T^{3}$. This case is convenient since we can work algebraically with functions which are finite linear combinations of the Fourier modes. But let us first briefly recall the construction in the case of $X=G$ based on loop group representations.

Fix a unitary highest weight representation of the central extension $\widehat{L G}$ of the smooth loop group $L G$ in a Hilbert space $V$. Use the standard 2-cocycle $k c$ with

$$
c(X, Y)=\frac{i}{2 \pi} \int_{S^{1}}<X, d Y>
$$

where $\langle\cdot, \cdot\rangle$ is an invariant bilinear form on the Lie algebra $\mathfrak{g}$ such that the level $k$ is a nonnegative integer in an irreducible highest weight representation of the loop group. In addition one needs an irreducible representation of the canonical anticommutation relations algebra

$$
\psi_{a}^{n} \psi_{b}^{m}+\psi_{b}^{m} \psi_{a}^{n}=2 \delta_{a b} \delta_{n,-m} \mathbf{1}
$$

in a Fock space $\mathcal{F}$. Here $n, m \in \mathbb{Z}$ and $a, b=1,2, \ldots \operatorname{dim} G$ relate to an orthonormal basis of $\mathfrak{g}$ with respect to $\langle\cdot, \cdot\rangle$. The representation is characterized by the existence of a vacuum vector with the property $\psi_{a}^{n} \mid 0>=0$ for $n<0$. In addition, we have the hermiticity relation $\left(\psi_{a}^{n}\right)^{*}=\psi_{a}^{-n}$.

Following the notation in [3] we define the skew symmetric operator

$$
Q=\sum \psi_{a}^{n}\left(T_{a}^{-n}+\frac{1}{3} K_{a}^{-n}\right)
$$

acting in the tensor product $V \otimes \mathcal{F}$. Here $S_{a}^{n}=T_{a}^{n} \otimes \mathbf{1}+\mathbf{1} \otimes K_{a}^{n}$ are the generators of the loop algebra acting in the tensor product. The commutators of the generators $K_{a}^{n}$ are determined by the central extension of level $k=\kappa$, where $\kappa$ is the dual Coxeter number of the simple Lie algebra $\mathfrak{g}$,

$$
\left[K_{a}^{n}, K_{b}^{m}\right]=\sum_{c} \lambda_{a b}^{c} K_{c}^{n+m}+\kappa m \delta_{a b} \delta_{n,-m}
$$

where the numbers $\lambda_{a b}^{c}$ are the structure constants of the Lie algebra $\mathfrak{g}$. In a unitary representation of the loop algebra $\left(T_{a}^{n}\right)^{*}=-T_{a}^{-n}$ and $\left(K_{a}^{n}\right)^{*}=-K_{a}^{-n}$.

Next define the family of operators $Q_{A}=Q+\tilde{k} \sum \psi_{a}^{n} A_{a}^{-n}$ where the $A_{a}^{n}$ 's are the Fourier components of the gauge potential $A$ in the Lie algebra basis labelled by $a$ and $\tilde{k}=k+\kappa$. One then shows that the family of operators $Q_{A}$ transforms covariantly under the central extension $\widehat{L G}$,

$$
\hat{g}^{-1} Q_{A} \hat{g}=Q_{A^{g}}
$$

with $A^{g}=g^{-1} A g+g^{-1} d g$ for $g \in L G$. Because of the central extension this family cannot be pushed down to a family of operators parametrized by $\mathcal{A} / L G$ but it 
defines a twisted equivariant $\mathrm{K}$ theory element on $G$. The Dixmier-Douady class of the twist is given by $\tilde{k}$ times the generator $\omega \in \mathrm{H}^{3}(G, \mathbb{Z}) \simeq \mathbb{Z}$.

\section{Gauge Current algebra in 3 Dimension}

Let $M$ be a compact connected spin 3-manifold, $G$ a compact simple Lie group and $\mathfrak{g}$ its Lie algebra. Let $E$ be a trivial vector bundle over $M$ with fiber the tensor product $\mathbb{C}^{2} \otimes \mathbb{C}^{N}$ where the first factor is the space of Weyl spinors and the group $G$ is unitarily represented in the second factor. The Weyl-Dirac operator $D_{A}$ coupled to a gauge potential $A$, with values in $\mathfrak{g}$, acts in a dense domain of the Hilbert space $H=L_{2}(M, E)$ of square integrable sections of the bundle $E$.

For a real number $\lambda$ and a given vector potential $A$ one has a polarization

$$
H=H_{+}(A, \lambda) \oplus H_{-}(A, \lambda)
$$

of the Hilbert space $H$ using the spectral projection associated to $D_{A}$ with a spectral cut at $\lambda$. This polarization defines a representation of the canonical anticommutation relations algebra (CAR) in a fermionic Fock space with a vacuum $\mid A, \lambda>$. The canonical anticommutation relations are generated by elements $a^{*}(v)$ (linear in $v \in H$ ) and $a(v)$ (antilinear in $v$ ) with relations

$$
a^{*}(u) a(v)+a(v) a^{*}(u)=2<v, u>\cdot \mathbf{1}
$$

where $\langle\cdot, \cdot\rangle$ is the $L_{2}$ inner product in $H$. The vacuum is a vector in the Fock space $\mathcal{H}$ such that

$$
a(v)\left|A, \lambda>=0=a^{*}(u)\right| A, \lambda>\text { for } v \in H_{+}(A, \lambda) \text { and } u \in H_{-}(A, \lambda) .
$$

However, there is a problem related to the spectral flow as $A$ varies which obstructs a construction of the Fock vacua as continuous functions of the argument $A$. To circumvent this problem one has to modify the construction by a certain family of local complex line bundles over open sets in the space $\mathcal{A}$ of smooth vector potentials. The group $\mathcal{G}$ of smooth gauge transformations does not act in these line bundles; instead, one has to construct an abelian extension $\hat{\mathcal{G}}$ which acts in the line bundles. The Lie algebra of the extension is defined by the 2-cocycle

$$
c(A ; X, Y)=\frac{1}{24 \pi^{2}} \int_{M} \operatorname{tr} A[d X, d Y]
$$

where $X, Y: M \rightarrow \mathfrak{g}$ and the trace is evaluated in the representation in $\mathbb{C}^{N}$. There are several ways to derive this formula but the index theory derivation in [1] describes best the topological and geometric aspects related to the construction of gerbes over the moduli space of gauge potentials.

As a consequence of the line bundle modifications is that the gauge group $\mathcal{G}$ acts through an extension $\hat{\mathcal{G}}$, infinitesimally determined by the cocycle $c$, on a vector bundle $V$ over $\mathcal{A}$ with model fiber $\mathcal{H}$.

Since $c$ is a function of $A$ the 2-cocycle property reads as

$$
\begin{aligned}
& c(A ; X,[Y, Z])+c(A ; Y,[Z . X])+c(A ; Z,[X, Y]) \\
& \quad+L_{X} c(A ; Y, Z)+L_{Y} c(A ; Z, X)+L_{Z} c(A, X, Y)=0
\end{aligned}
$$

where $L_{X}$ denotes the Lie derivative acting on functions of $A$ through the infinitesimal gauge transformations $L_{X} A=[A, X]+d X$. Alternatively, the cocycle $c$ comes 
from a central extension of the gauge groupoid $(\mathcal{A}, \mathcal{G})$ with sources and targets in $\mathcal{A}$ and arrows $A \rightarrow A^{g}=g^{-1} A g+g^{-1} d g$.

Remark In a real representation of $\mathfrak{g}$ the cocycle $c$ vanishes identically. The reason is that in a real unitary representation the Lie algebra elements are antisymmetric and therefore $\operatorname{tr} A(B C+C B)=\operatorname{tr}(A(B C+C B))^{t}=-\operatorname{tr}(B C+C B) A=$ $-\operatorname{tr} A(B C+C B)=0$. In particular, this is always the case for the adjoint representation of $\mathfrak{g}$.

The above remark is important in the next section. We shall consider Fock representations of the current algebra in the tensor product of two Fock spaces. The first one comes from the quantization of fermions in the adjoint representation and the second in an arbitrary complex representation. It follows that the 2-cocycle $c$ has a contribution only from the complex representation.

\section{FAMILY OF FORMAL SUPERSYMMETRIC HAMILTONIANS}

Using the symmetric bilinear form

$$
<X, Y>=\frac{1}{(2 \pi)^{3}} \int \operatorname{tr} X(z) Y(z) d z
$$

on the algebra of smooth functions $X, Y: T^{3} \rightarrow \mathfrak{g}$ we associate to the Fourier modes $T_{a}^{n}\left(n \in \mathbb{Z}^{3}\right.$ and $\left.a=1,2, \ldots N=\operatorname{dim} \mathfrak{g}\right)$ the dual basis $\psi_{a}^{n}$ and define the formal series

$$
Q=\sum_{n \in \mathbb{Z}^{3}} \sum_{a=1}^{N}\left(\psi_{a}^{n} T_{a}^{-n}+\frac{1}{3} \psi_{a}^{n} K_{a}^{-n}\right)
$$

The vectors $\psi_{a}^{n}$ are interpreted as elements in an infinite dimensional Clifford algebra with anticommutation relations

$$
\psi_{a}^{n} \psi_{b}^{m}+\psi_{b}^{m} \psi_{a}^{n}=2 \delta_{a b} \delta_{n,-m}
$$

Here the $K_{a}^{n}$ 's are derivations of the CAR algebra defined as

$$
\left[K_{a}^{n}, \psi_{b}^{m}\right]=\sum_{c} \lambda_{a b}^{c} \psi_{c}^{n+m}
$$

The commutators of the derivations and are then given as

$$
\left[K_{a}^{n}, K_{b}^{m}\right]=\sum_{c} \lambda_{a b}^{c} K_{c}^{n+m} .
$$

As infinite formal sums,

$$
K_{a}^{n}=-\sum_{b, c, m} \frac{1}{4} \lambda_{a b}^{c} \psi_{b}^{m} \psi_{c}^{n-m} .
$$

More concretely, in the case of Weyl fermions in the adjoint representation of the gauge group $G$, after quantization in the bundle of fermionic Fock spaces $\mathcal{F}$ over $\mathcal{A}$ the current algebra generated by the $K_{a}^{n}$ 's acts without the abelian extension $(c=0)$ by the remark in the end of the previous Section. For this reason it is actually convenient to deal with massive 4-component Dirac fermions instead of 2-component Weyl fermions. This simplifies some things later on. For massive fermions we have a mass gap in the spectrum of the Dirac hamiltonians and we can define the vacuum with respect to the polarization defined by the spectral cut at zero for all Dirac hamiltonians coupled to vector potentials. The fermionic 
modes $\psi_{a}^{n}$ can stand for any of the four components of the Dirac spinor. For notational simplicity we shall suppress the spinor label $\alpha=1,2,3,4$ in the following discussion.

The current algebra extension defined by the cocycle $c$ comes entirely from the action of the $T_{a}^{n}$ 's on the sections of the bundle $V$ over $\mathcal{A}$.

Since the gauge group acts in the fermionic Fock spaces $\mathcal{F}$ without the extension term, the Dixmier-Douady class of the projective Fock bundle vanishes and thus can be pushed to a true Hilbert bundle over the moduli space $\mathcal{A} / \mathcal{G}_{0}$, the sections of this bundle corresponding to gauge invariant sections of the pull-back bundle over $\mathcal{A}$. In particular, we are free to define a gauge covariant vacuum section $A \mapsto|A\rangle$ with $g|A>=| g \cdot A>$. For an arbitrary polynomial $P$ in the generators $\psi_{a}^{n}$ the action of a gauge transformation on the state $P \mid A>$ is then given by the canonical action on the generators of the Clifford algebra (infinitesimally by the formula 3.2) and by $g \mid A>)=\mid g \cdot A>$.

As it stands, the infinite sum in 3.1 is ill-defined as an operator but $Q$ defines a derivation of the CAR algebra and the gauge current algebra as

$$
\begin{aligned}
{\left[Q, \psi_{b}^{m}\right]_{+} } & =2\left(T_{b}^{m}+K_{b}^{m}\right) \equiv 2 S_{b}^{m} \\
{\left[Q, S_{b}^{m}\right] } & =\sum_{n, a} c\left(A ; T_{a}^{n}, T_{b}^{m}\right) \psi_{a}^{-n}
\end{aligned}
$$

For a smooth potential $A$ the infinite sum in the second equation is actually converging in the $L^{2}$ norm in the CAR algebra generated by the $\psi_{a}^{n}$ 's since the Fourier coefficients of a smooth function form a rapidly decreasing sequence.

Remark An $N \times N$ matrix $X$ defines an element $\psi(X)$ in the dual $\mathfrak{g}^{*}$ by $\langle\psi(X), z>=\operatorname{tr} X z$ where $z$ stands for the matrix representing $z \in \mathfrak{g}$ in the representation space $\mathbf{C}^{N}$. In the same vain, a matrix valued 3 -form $\omega$ on $T^{3}$ defines an element in the dual of the current algebra $\operatorname{Map}\left(T^{3}, \mathfrak{g}\right)$ by

$$
<\psi(\omega), X>=\int \operatorname{tr} \omega X .
$$

It follows that for a linear combination $X=\sum X_{m}^{a} S_{a}^{m}$ we have

$$
[Q, X]=\psi(d X \wedge d A+d A \wedge d X) .
$$

From (3.3) (3.4) one can reduce that the commutators with the formal square $Q^{2}$ are given as

$$
\begin{aligned}
& {\left[Q^{2}, \psi_{a}^{n}\right]=2 \psi_{b}^{m} c\left(A ; T_{b}^{-m}, T_{a}^{n}\right)} \\
& {\left[Q^{2}, S_{a}^{n}\right]=2 S_{b}^{m} c\left(A ; T_{b}^{-m}, T_{a}^{n}\right)-\psi_{b}^{m} \psi_{c}^{p} \mathcal{L}_{c}^{-p} c\left(A ; T_{b}^{-m}, T_{a}^{n}\right) .}
\end{aligned}
$$

We have used the fact that

$$
\sum_{b} \mathcal{L}_{b}^{p} c\left(A ; T_{b}^{-p}, T_{a}^{n}\right)=0
$$

which follows from

$$
\mathcal{L}_{Z} c(A ; X, Y)=\int \operatorname{tr}[A, Z](d X d Y-d Y d X)
$$

and the algebraic relation

$$
\sum_{b} \operatorname{tr}\left[A, T_{b}\right]\left(T_{b} T_{a}+T_{a} T_{b}\right)=\sum_{b} \operatorname{tr}\left[T_{a}, A\right]\left(T_{b}\right)^{2}=\sum_{b} \operatorname{tr} T_{a}\left[A,\left(T_{b}\right)^{2}\right]=0
$$


since the element $\sum_{b}\left(T_{b}\right)^{2}$ is the Casimir invariant in the semi simple Lie algebra $\mathfrak{g}$.

The formal square of $Q$ can be written as

$$
Q^{2}=\sum T_{a}^{n} T_{a}^{-n}+\frac{1}{2} \sum c\left(A ; T_{a}^{n}, T_{b}^{m}\right) \psi_{a}^{n} \psi_{b}^{m}
$$

and one check by direct computation from the commutation relations that the commutators of this formal series match the commutators (3.5) and (3.6). This expression can be compared with the corresponding formula [3] in the loop group case. The important difference is that the contribution involving the dual Coxeter number is missing. The reason is that the algebra of the $K_{a}^{n}$ 's does not contain the anomaly proportional to the dual Coxeter number.

From (3.3) follows immediately

Theorem 3.1. Let $B=B(A)$ be a matrix value 3-form on $T^{3}$ defined as

$$
B(A)=\omega \wedge d A+d A \wedge \omega+\alpha \wedge A \wedge \beta+\gamma \wedge A+A \wedge \phi+\theta
$$

where the parameters $\omega, \alpha, \beta$ are matrix valued 1-forms, $\gamma, \phi$ are matrix valued 2forms and $\theta$ is a matrix valued 3-form. Thus $B(A)$ is an affine function in the variable $A$. Define the formal sum $Q_{B}=Q+\psi(B(A))$. Then for $X=\sum X_{m}^{a} S_{a}^{m}$

$$
\left[Q_{B}, X\right]=\psi\left(B^{\prime}(A)\right)
$$

where $B^{\prime}(A)$ is again a matrix valued 3-form with new parameters $\left(\omega^{\prime}, \alpha^{\prime}, \beta^{\prime}, \gamma^{\prime}, \phi^{\prime}, \theta^{\prime}\right)$. In particular,

$$
\omega^{\prime}=[\omega, X]+d X
$$

Thus $\omega$ transforms like a matrix valued vector potential under the infinitesimal gauge transformations $X$. In particular, the action of based gauge transformations is free on the parameter $\omega$ and thus also on the space of functions $A \mapsto B(A)$,

Corollary 3.2. The moduli space $\mathcal{B} / \mathcal{G}_{0}$ of the functions $B(A)$ modulo the group $\mathcal{G}_{0}$ of based gauge transformations is the classifying space for the group $\mathcal{G}_{0}$ and thus homotopy equivalent to the space $\mathcal{A} / \mathcal{G}_{0}$ of gauge equivalence classes of vector potentials $A$.

The forms $Q_{B}$ are only formal algebraic expressions but they can be used to define sesquilinear forms in dense domains of the Fock spaces. Let $\xi$ be an arbitrary smooth section of $V$ and $\eta(A)=\mid A>$ the vacuum section of $\mathcal{F}$. The domain $D$ for $Q_{B}$ is then defined to consists of sections of $V \otimes \mathcal{F}$ of the form $\xi \otimes p(\psi) \eta$ where $p$ is a polynomial in the generators $\psi_{a}^{n}$ of the Clifford algebra.

Proposition 3.3. With the notation above $<\xi^{\prime} \otimes p^{\prime}(\psi) \eta, Q_{B}(\xi \otimes p(\psi) \eta>$ is welldefined in the fiberwise inner product for the sections.

Proof. The vacuum section $\eta$ is invariant under gauge transformations so $K_{a}^{n} \eta=0$ and thus also $\sum \psi_{a}^{n} K_{a}^{-n} \eta=0$ and so $Q(\xi \otimes \eta)=\sum T_{a}^{n} \xi \otimes \psi_{a}^{-n} \eta$. Using the algebra relations (3.3), (3.4) one sees that it is sufficient to show that the inner products $<\xi^{\prime} \otimes p^{\prime} \eta, T_{a}^{n} \xi \otimes \psi_{a}^{-n} p \eta>$ exist fiberwise. First computing this at the fiber over $A=0$ the claim follows from the fact that for large enough absolute value of $n$ the inner product of $\psi_{a}^{n}$ between finite polynomials in the $\psi$ 's vanish. This is a consequence of the fact that the free vacuum is an eigenvector for the quantized 
momentum vectors $P_{k}$ with eigenvalue zero and thus the vacuum expectation value of a polynomial not commuting with the $P_{k}$ 's is equal to zero.

When $A \neq 0$ the above argument needs a modification.

To be more precise on the definition of the momentum operators: These are not the free momentum operators but momenta defined with respect to the vacuum $\mid A>$. That is, choosing a unitary operator $T_{A}$ in the 1-particle Hilbert space such that $T_{A}^{-1} \epsilon_{0} T_{A}=\epsilon_{A}$ where $\epsilon_{A}=D_{A} /\left|D_{A}\right|$ then $p_{k}=T_{A}^{-1} i \frac{\partial}{\partial x_{k}} T_{A}$ in the 1-particle space and $P_{k}$ is the second quantized operator corresponding to $p_{k}$. Here $T_{A}$ is a pseudodifferential operator with an asymptotic expansion $T_{A}=1+t_{-1}+t_{-2}+\cdots$ in inverse powers of momenta; the first nontrivial term $t_{-1}$ is linear in $A$, [7. It follows that $p_{k}=i \frac{\partial}{\partial x_{k}}+$ terms of order -1 in momenta. Now the commutator $\left[P_{k}, \psi_{a}^{n}\right]$ is note any more equal to $n_{k} \psi_{a}^{n}$ but there is correction which is proportional to the inverse of $|n|$. It follows that the expectation value of $\psi_{a}^{n}$ between polynomial states in the Clifford algebra is not exactly zero for large $|n|$ but there is a correction proportional to $1 /|n|^{2}$ (since the extra terms in $P_{k}$ have relative magnitude of order -2$)$. But the factor $1 /|n|^{2}$ guarantees that the sum over $n$ in $\sum \psi_{a}^{n} T_{a}^{-n}$ gives a finite fiberwise expectation value between sections of the form $\xi \otimes p(\psi) \eta$. The second term $\psi_{a}^{n} K^{-n}$ gives a finite contribution by $K^{n}{ }_{a} \eta=0$ and the relation (3.3). The interaction term $\psi(B(A))$ is in fact a fiberwise well-defined operator since it is a linear combination of the $\psi_{a}^{n}$ 's with $L_{2}$ coefficients coming from a smooth form $B(A)$.

\section{REFERENCES}

[1] Carey, Alan; Mickelsson, Jouko; Murray, Michael; Index theory, gerbes, and Hamiltonian quantization. Comm. Math. Phys. 183 (1997), no. 3, 707 - 722.

[2] Freed, Daniel S.; Hopkins, Michael J.; Teleman, Constantin: Loop groups and twisted Ktheory III. Ann. of Math. (2) 174 (2011), no. 2, 947 - 1007.

[3] Mickelsson, Jouko: Gerbes, (twisted) K-theory, and the supersymmetric WZW model. Infinite dimensional groups and manifolds, 93 - 107, IRMA Lect. Math. Theor. Phys., 5, de Gruyter, Berlin, 2004.

[4] Douglas, Christopher L: On the twisted K-homology of simple Lie groups. Topology 45 (2006), no. $6,955-988$.

[5] Mickelsson, Jouko: Chiral anomalies in even and odd dimensions. Comm. Math. Phys. 97 (1985), no. 3, $361-370$.

[6] Mickelsson, Jouko: Kac-Moody groups, topology of the Dirac determinant bundle, and fermionization. Comm. Math. Phys. 110 (1987), no. 2, 173 - 183.

[7] Mickelsson, Jouko: Wodizicki residue and anomalies of current algebras. In: Springer Lecture Notes in Physics 436, "Integrable Models and Strings", pp. 123 - 135.

[8] Faddeev, L. D.; Shatashvili, S. L.: Algebraic and Hamiltonian methods in the theory of nonabelian anomalies. (Russian) Teoret. Mat. Fiz. 60 (1984), no. 2, 206 - 217.

Department of Mathematics and Statistics, University of Helsinki

E-mail address: jouko@kth.se 